\title{
QUALIDADE DA INFORMAÇÃO CONTÁBIL DAS SOCIEDADES DE ECONOMIA MISTA: UMA COMPARAÇÃO ENTRE O PERIODO PRÉ E PÓS ADOÇÃO DAS IFRS
}

\author{
Quality of the accounting information of the semi-state society - a \\ comparison between the period before and after the adoption of IFRS
}

\author{
Carlos Alberto Kühl'; Marcos Roberto Kühl²
}

\begin{abstract}
${ }^{1}$ Doutor em Controladoria e Contabilidade. Professor do Departamento de Ciências Contábeis da Universidade Estadual do Centro-Oeste, UNICENTRO - Guarapuava - Paraná. E-mail: ckuhl@ unicentro.br

${ }^{2}$ Doutor em Administração. Professor do Departamento de Ciências Contábeis e do Programa de PósGraduação em Administração da Universidade Estadual do Centro-Oeste, UNICENTRO - Guarapuava - Paraná.E-mail: mkuhl@unicentro.br
\end{abstract}

Data do recebimento: 25/03/2021 - Data do aceite: 17/06/2021

RESUMO: A Sociedade de Economia Mista (SEM) é um tipo societário sui generis que não tem merecido atenção especial nos estudos, principalmente nos que são relacionados com a Ciência Contábil. A particularidade de possuir aspectos públicos e particulares, função econômica e social e de estar intimamente atrelada aos aspectos políticos e partidários, pode aduzir que essas sociedades não necessitam apresentar níveis de qualidade da informação contábil compatíveis com os outros tipos societários. Nesse contexto, o objetivo deste artigo é analisar se a qualidade da informação contábil evidenciada pelas SEM sofreu alteração após a harmonização contábil brasileira com as normas internacionais do IASB. Para a consecução do objetivo, foram avaliadas 141 SEM durante o período de 1995 a 2014 em dois constructos de qualidade da informação contábil (persistência dos resultados e conservadorismo condicional), através de regressões em dados em painel e em corte transversal. Os resultados indicam que a qualidade da informação contábil das SEM não apresenta alteração significativa após a adoção das IFRS, no Brasil, quando analisado o constructo de Conservadorismo Condicional. Já para o constructo de Persistência dos Resultados apresenta uma variação indicando que os resultados contábeis são mais persistentes após a adoção as IFRS. 
Palavras-chave: Sociedades de Economia Mista. Qualidade da Informação Contábil. Persistência dos Resultados. Conservadorismo Condicional.

\begin{abstract}
The Semi-State Society (SSS) is a unique type of company that has not earned special attention in studies, especially those related to accounting science. The particularity of having public and private aspects, economic and social function and of being linked to the political and partisan aspects, can adduce that these societies do not need to present levels of quality of the accounting information compatible with other corporate types. In this context, the purpose of this article is to examine whether the quality of the accounting information evidenced by the unchanged after the Brazilian accounting harmonization with the international standards of the IASB. To achieve the goal, 141 SSS were evaluated during the period from 1995 to 2014 in two quality constructs of accounting information (persistence of results and conditional conservatism), through regressions in panel data and cross-sectional. The results indicate that the quality of the accounting information of the SSS does not present a significant change after the adoption of IFRS in Brazil when analyzed the construct of conditional conservatism. For the construct of persistence of the results, it presents a variation indicating that the accounting results are more persistent after the adoption of IFRS.
\end{abstract}

Keywords: Semi-State Society. Quality of Accounting Information. Persistence of Results. Conditional Conservatism.

\section{Introdução}

O ambiente empresarial brasileiro é bem diverso em relação às formas de constituição de empresas e permite inúmeras configurações de quadro social ou de acionista, com o intuito de formar um patrimônio empresarial.

Uma das formas de configuração de acionistas é aquela que congrega recursos públicos, oriundos de um ente governamental com recursos privados, oriundos de pessoas físicas ou jurídicas e que recebe por parte da legislação brasileira a alcunha de Sociedade de Economia Mista (SEM).

As SEM brasileiras são, para grande parte da população, empresas que pertencem ao Estado e que devem executar as suas atividades com o menor ônus possível para o cidadão, ou seja, cumprir o seu papel social frente à sociedade, mesmo com a eventual perda de rentabilidade ocasionada pelo cumprimento desse papel social. (BIN; CASTOR, 2007). A qualidade das informações contábeis das SEM é importante para a população, pois auferirá a credibilidade da administração dos recursos públicos nelas investidos e evitando prejuízos futuros com sua alocação para fins espúrios, servindo como uma forma de prestação de contas (accountability) dos administradores e podendo ser utilizada para mensurar as diferentes formas de gerenciamento dos resultados das SEM.

A partir do ano 2010 as empresas brasileiras foram obrigadas a apresentar as suas demonstrações contábeis em consonância com as normas internacionais, também conhecidas como IFRS, emanadas pelo International 
Accounting Standards Board, as quais, segundo alguns estudos (FERREIRA; LEMES; FORTI, 2015; DAMASCENA; DUARTE; PAULO, 2017) tenderiam a apresentar uma qualidade da informação contábil reportada mais avançada da que era apresentada pelas normas e regras anteriores.

Diante disto, a questão que permeia a presente pesquisa é se no ambiente das SEM a adoção dos normas internacionais (IFRS) apresentaram impacto na qualidade da informação contábil, analisada pelos constructos de persistência dos resultados e de conservadorismo condicional, ou seja, houve alteração na qualidade da informação contábil reportada pelas SEM após a adoção das IFRS no Brasil?

O artigo está estruturado em quatro tópicos além desta introdução, sendo o primeiro destinado à apresentação do referencial teórico dos conteúdos; no segundo são descritos os aspectos metodológicos utilizados, em especial a descrição dos modelos econométricos utilizados. No terceiro tópico são apresentados e discutidos os resultados dos modelos econométricos e o último tópico destina-se às considerações finais sobre os achados da pesquisa.

\section{Referencial teórico}

Na conceituação de Mello (2007), a Sociedade de Economia Mista é um mero instrumento de ação do Estado, para implementar objetivos que assumiu no interesse da coletividade, ou seja, é um braço da Estado com a participação dos recursos privados. Considerando a sua definição, as características principais de uma SEM são: a) a associação de capital público e privado; b) a direção da empresa por ambos os investidores; c) o interesse público que tal sociedade visa; e d) a sua criação que é sempre estabelecida por lei. (BONFIM, 2011).
Essas características permeiam a complexidade das SEM, pois, elas tentam congregar interesses antagônicos dos capitais privados e públicos, sendo que os primeiros aspiram por questões microeconômicas e os outros, por questões macroeconômicas. (RUCKERT, 1981).

Entretanto, para a esfera contábil e com base no texto constitucional as SEM devem obrigatoriamente sujeitar-se ao regime jurídico próprio das empresas privadas, estando incurso nesta sujeição a elaboração da contabilidade nos moldes vigentes às demais sociedades empresariais. (MORAES, 2002).

A partir dessa previsão legal, as SEM devem elaborar as demonstrações financeiras em consonância com a legislação vigente no País e essas devem "fornecer informações financeiras sobre a entidade que reporta que sejam úteis para investidores, credores por empréstimos e outros credores, existentes e potenciais, na tomada de decisões referente à oferta de recursos à entidade." (CPC, 2019, p.5).

Considerando isso, em que pese a ambiguidade de objetivos, as SEM podem ser alvo de análise da qualidade dos resultados reportados, pois a sua contabilidade deve ser baseada nos mesmos princípios e normas descritos para as demais sociedades empresariais e deve ser utilizada para mitigar os problemas oriundos da teoria da agência e para aprimorar a governança corporativa dessas entidades.

$\mathrm{Na}$ visão de Ewert e Wagenhofer (2011, p.115) “a 'verdadeira' qualidade da informação contábil não é observável. Para isto diversos estudos empíricos procuram utilizar-se de métricas para avaliar a qualidade", como pode ser verificado nos trabalhos de Basu (1997), Dechow e Skinner (2000), Watts (2003), Schipper e Vicent (2003) entre outros.

O trabalho de Dechow, Ge e Schrand (2010) elabora um levantamento das pes- 
quisas realizadas sobre a qualidade da informação contábil e sistematiza os resultados, apresentando as conclusões e os aspectos positivos e negativos, destacados pelas pesquisas, salientando que elas procuram estabelecer e testar métricas que possam ser consideradas válidas para poder avaliar a qualidade das informações contábeis. As autoras ainda afirmam que as variáveis contábeis utilizadas para a mensuração da qualidade da informação contábil deveriam ser persistentes, conservadoras e sem gerenciamento de resultados.

A persistência é uma das qualidades da informação contábil, descrita por Palepu, Healy e Bernard (2004) que afirmam que o enorme interesse pela persistência dos resultados contábeis está relacionado com a sua característica de predição, pois essa característica auxilia na avaliação dos ativos das entidades.

A teoria da persistência dos resultados contábeis realça que empresas com resultados mais persistentes tem um fluxo mais sustentável de lucros ou fluxos de caixa, tornando a informação mais útil para a tomada de decisão e, dessa forma, quanto maior a persistência do resultado contábil maior a qualidade da informação reportada. (DECHOW, GE; SCHRAND, 2010).

Para a análise da qualidade da informação contábil, se ela está evidenciada sem vieses de manipulação ou gerenciamento, pode-se inferir que o lucro de amanhã é uma função do lucro de hoje (SLOAN, 1996) e a persistência dos resultados contábeis poderá ser evidenciada.

Já o conservadorismo foi considerado por Sterling (1970) como o mais influente princípio contábil, devendo ser entendido como uma reação prudente frente à incerteza, em consonância com o que dispunha o Statement of Financial Accounting Concepts $\mathrm{n}^{\mathrm{o}}$ 02, emitido pelo FASB (Financial Accounting
Standards Board), de que o conservadorismo deve utilizar-se da estimativa menos otimista, quando da existência de mais de uma estimativa válida. (FASB, 1975).

$\mathrm{Na}$ mesma linha de raciocínio, Lopes (2002) o descreve como uma das características mais importantes do corpo de práticas e procedimentos realizados pela contabilidade e estava presente na maioria das estruturas conceituais emitidas pelos órgãos responsáveis pela emissão de normas, tanto internacionalmente quanto no modelo normativo brasileiro.

Na visão de Coelho (2007, p.56) “(...) a maioria dos códigos de princípios contábeis exarados mundialmente tratam o conservadorismo na ótica de lançar valores com parcimônia, pessimistamente, sem estabelecer um propósito ou uma lógica que não o costume ou a tradição como justificativa para esse procedimento".

Em termos contábeis o conservadorismo ou prudência, estabelece que havendo possibilidades alternativas de avaliação do patrimônio, deve haver a opção por aquela que represente o menor valor para os itens de ativo e receita e o maior valor para os itens do passivo e da despesa. (MOST, 1982).

$\mathrm{Na}$ esfera econômica, nas palavras de Lopes (2002) a prudência pode ser entendida como a incorporação enviesada pela contabilidade da realidade econômica.

Para Bliss (1924, apud BASU, 1997), os contadores expressam o conservadorismo utilizando a regra de não antecipar lucros e antecipar todas as perdas, destacando que o resultado econômico pode ser enviesado mais pelas más notícias do que pelas boas notícias.

No entendimento de Paulo, Antunes e Formigoni (2008, p.51),

Devido à presença do conservadorismo nos números contábeis, há uma tendência de maior persistência dos ganhos do que das perdas. O conservadorismo faz, portanto, 
com que as acumulações (accruals) sejam assimétricas, visto que as perdas tendem a ser totalmente reconhecidas, ao passo que o mesmo não ocorre com os ganhos.

Basu (1997, p. 7) assim define o conservadorismo:

Eu interpreto esta regra como denotando a tendência dos contadores em requerer um grau de verificação maior para reconhecer boas notícias como os ganhos do que reconhecer más notícias como as perdas; [...] conservadorismo resulta em grande probabilidade de reconhecimento contábil tempestivo das más notícias do que das boas notícias.

Nas pesquisas, o conservadorismo é analisado sob duas perspectivas: a condicional e a incondicional. Coelho (2007, p. 136) conceitua as duas formas de conservadorismo da seguinte maneira:

a) Incondicional, decorrente da regra de que entre duas alternativas de mensuração e reconhecimento de eventos, igualmente válidas, deve-se escolher aquela que resulte na menor avaliação do patrimônio dos proprietários; seu propósito é o da prudência e está relacionado ao grau de incerteza sobre os efeitos derivados de transações iniciadas;

b) Condicional, que se refere à possibilidade de antecipar o reconhecimento contábil de perdas econômicas - portanto, ainda não realizadas - à luz de estimativas realizadas pela gerência com base em eventos atuais de cunho negativo; note-se que esta forma equivale a reconhecer fatos econômicos na contabilidade com oportunidade, de maneira assimétrica, privilegiando os indícios de resultados negativos.

Na interpretação de Beaver e Ryan (2005), o conservadorismo incondicional já está incorporado nas operações normais da empresa e o conservadorismo condicional é dependente de uma notícia anormal às operações da empresa e será incorporada a contabilidade como respostas dos administradores a essas notícias.

Em consequência disso, uma "característica de alta qualidade dos resultados é a proporção de perdas reconhecidas no resultado quando elas ocorrem em contraponto ao diferimento para períodos futuros". (RODRIGUES, 2012, p. 43).

O que remete a interpretação geral de que quanto mais conservador o resultado reportado, maior será a qualidade deste resultado, pois reconhecerá antecipadamente as perdas econômicas.

Basu (1997) apresentou em seus estudos uma métrica que permite mensurar o conservadorismo condicional, a qual é uma das mais utilizadas pela literatura contábil sobre o assunto.

A equação básica proposta pelo modelo de Basu (1997) é

$\frac{X_{i t}}{P_{i t-1}}=\alpha_{0}+\alpha_{1} D R_{i t}+\beta_{0} R_{i t}+\beta_{1} R_{i t} * D R_{i t}+\varepsilon_{i t}$

A equação regride as mudanças de resultados ponderando-as pelos preços das ações no início do período, sobre mudanças dos resultados positivos e das mudanças dos resultados negativos. O retorno econômico da ação é uma proxy para o resultado econômico, baseado em algum nível de eficiência de mercado. A regressão pode analisar se o resultado contábil incorpora, significativamente, o resultado econômico (significância do coeficiente ), avaliando, assim, a oportunidade (tempestividade) do seu reconhecimento contábil. (PAULO, ANTUNES; FORMIGONI, 2008).

Em relação a qualidade da informação contábil após a convergência ao IFRS os estudos de Santos e Cavalcante (2014); Castro et al. (2015) e Sousa, Souza e Demonier (2016), 
atestaram não haver alteração significativa na qualidade da informação contábil reportada após a adoção dos IFRS, em contraponto a isto as pesquisa de Silva, Bonfim e Niyama (2017) e Silva, Brighenti e Klann (2018) concluíram que existem indícios de alteração na qualidade da informação contábil, para mais elevada, após a adoção dos IFRS. Salienta-se a ressalva de Silva, Bonfim e Niyama (2017) que para empresas que estão inseridas em atividades reguladas, os resultados não são conclusivos.

\section{Aspectos metodológicos}

A pesquisa tem caráter hipotético-dedutivo, buscando inferências a partir de dados de amostra de demonstrações contábeis das Sociedades Anônimas e de informações adicionais coletadas nos relatórios de administração das Sociedades de Economia Mista. As inferências estatísticas serão realizadas com base em modelos de regressão com dados em painel.

O objetivo principal da pesquisa é analisar se houve alteração na qualidade da informação contábil evidenciada pelas Sociedades de Economia Mista após a adoção das IFRS pela contabilidade brasileira.

\section{Dados e amostra}

Os dados primários para a pesquisa foram coletados junto ao banco de dados do projeto Melhores e Maiores, vinculado a FIPECAFI - Fundação Instituto de Pesquisas Contábeis, Atuariais e Financeiras, complementadas por informações disponíveis no site da Comissão de Valores Mobiliários e nos endereços eletrônicos das empresas.

As informações analisadas compreendem o período de 1995 a 2016, sendo que os anos de 2015 e 2016 foram excluídos das análises em virtude de que algumas das Sociedades de Economia Mista constantes do banco de dados foram citadas nas investigações no âmbito da Operação Lava Jato, os impactos destas investigações ainda não foram devidamente apurados e analisados e reconhecidos nos demonstrativos contábeis.

\section{Procedimentos estatísticos}

Para a análise dos dados das empresas foram efetuadas estimações de regressões com dados em painel para avaliar a qualidade da informação contábil das sociedades de economia mista especificamente nos constructos de persistência dos resultados contábeis e conservadorismo, considerando a disponibilidade das informações os painéis de dados utilizados são desbalanceados, ou seja, não apresentam informações de todas as empresas para todo o período de análise, possuindo assim lacunas de dados faltantes.

Os dados em painel consideram que as entidades podem possuir características próprias e diferenciadoras e que essas podem ser constantes ao longo do tempo, tal heterogeneidade não é levada em consideração em estudos que se utilizam de dados em séries temporais ou em cortes transversais e os resultados apurados por estas sistemáticas podem produzir resultados enviesados.

Além da vantagem citada anteriormente, Baltagi (2008) descreve outras vantagens da utilização de dados em painel em relação aos cortes transversais e séries temporais e em suma, de acordo com Gujarati e Porter (2011, p.589), “os dados em painel podem enriquecer a análise aplicada do ponto de vista de ser impossível usarmos apenas dados de séries temporais ou de corte transversal".

De acordo com Fávero et al. (2009), as abordagens mais comuns de análise de dados em painel são: a POLS que considera o beta de uma variável explicativa igual para todas as observações ao logo do tempo; Efeitos Fixos que considera as alterações em cada 
observação ao longo do tempo; e a abordagem de Efeitos Aleatórios que refletem diferenças do intercepto de cada empresa no termo do erro.

Para a escolha da abordagem mais adequada para cada regressão são aplicados os testes $F$ de Chow, LM de Breusch e Pagan e $W$ de Hausman, de acordo com a literatura sobre o assunto (BALTAGI, 2008; GUJARATI; PORTER, 2011).

Dessa forma, para as estimações dos constructos de persistência e conservadorismo foram utilizados os modelos de POLS e Efeitos Fixos, com a utilização da correção robusta dos erros-padrão. A utilização de um modelo de regressão robusta tem por finalidade deixar os coeficientes consistentes em relação à heterocedasticidade, corrigindo os erros-padrão dos coeficientes, que passam a ser denominados de erros-padrão robusto. (GUJARATI, 2006).

Considerando a necessidade de algumas correções, os modelos foram estimados com a matriz de erros-padrão robusto de Newey-West para correção de heterocedasticidade e autocorrelação.

\section{Descrição dos modelos econométricos}

Para a consecução do objetivo proposto, os dados contábeis das Sociedades de Economia Mista foram analisados, sob o prisma dos constructos de qualidade da informação contábil.

O modelo proposto por Dechow e Schrand (2004) para verificar a persistência dos resultados contábeis que foi utilizado no estudo está descrito na equação I:

Equação I - Modelo de Persistência utilizado

$$
X_{i t}=\beta_{0}+\beta_{1} X_{i t-1}+\epsilon_{i t}
$$

No modelo original descrito, quanto maior e mais próximo de um (1) for o coeficiente, com significância estatística, mais persistentes são os resultados contábeis no futuro. (DECHOW; SCHRAND, 2004).

Já o modelo original de observação do conservadorismo condicional (BASU, 1997) determinou o estabelecimento da relação entre os resultados contábeis negativos e o reconhecimento de perdas conservadoras para proteger os resultados contábeis futuros. (RODRIGUES, 2012).

Nesse modelo, umas das variáveis é o valor de mercado das ações da empresa, considerando a estrutura das entidades a serem analisadas pela pesquisa, faz-se necessário utilizar-se do modelo alternativo, implementado por Ball e Shivakumar (2005).

Considerando os pressupostos do estudo e de que se pretende analisar o nível de conservadorismo em diferentes grupos de entidades, o modelo econométrico geral é dado por:

\section{Equação II - Modelo de Conservadorismo utilizado}

$\Delta N I_{i t}=\begin{gathered}\alpha_{0}+\alpha_{1} D \Delta N I_{i t-1}+\alpha_{2} \Delta N I_{i t-1}+\alpha_{3} D \Delta N I_{i t-1} \\ D \Delta N I_{i t-1}+\alpha_{6} D U M M Y\end{gathered}$

A sistemática utilizada para avaliar o conservadorismo condicional será semelhante à utilizada nos trabalhos de Dai e Ngo (2013) e Bu, Zhang, Lin e Hu (2020), adaptada às particularidades das informações disponíveis e do objetivo proposto para o estudo.

\section{Resultados e análise dos dados}

A Tabela I apresenta a análise do constructo de persistência dos resultados contábeis, denotando que existe diferença estatística entre a persistência do período pré-adoção das IFRS e do período pós-adoção. 


\begin{tabular}{cccc}
\hline & $X_{i t}=\beta_{0}$ & $+\beta_{1} X_{i t-1}+\beta_{2} I F R S+\beta_{3} X_{i t-1} *$ IFRS $+\epsilon_{i t}$ \\
\hline Variável & Coeficiente & p-valor \\
\hline$\beta_{0}$ & $-0,00640$ & $0.006 * * *$ \\
$\beta_{1}$ & 0,03467 & 0,326 \\
$\beta_{2}$ & 0,12018 & $0.098 *$ \\
$\beta_{3}$ & 0,02348 & 0,507 \\
Observações & & 1.640 & \\
\hline
\end{tabular}

Nota. Método de estimação: Dados em Painel - Modelo de Efeitos Fixos com Erro Padrão Robusto; e são os lucros (resultados) das Sociedades de Economia Mista, escalonados pelo valor de seus ativos no início do ano; IFRS é a variável dummy que assume o valor 0 para os anos de 1995 a 2009 e o valor 1 para os anos de 2010 a 2014; é o termo de erro do modelo.

$*, * *$ e *** denotam significância estatística a $10 \%, 5 \%$ e $1 \%$, respectivamente.

O coeficiente apresentado na Tabela I é positivo e apresenta significância estatística, a 10\%, indicando que a persistência dos resultados no período pós-adoção das IFRS é maior do que o apresentado no período pré-adoção das IFRS, ou seja, no período pós-adoção os resultados de amanhã são explicados de forma mais incisiva pelos resultados atuais.

Tabela II - Conservadorismo Condicional

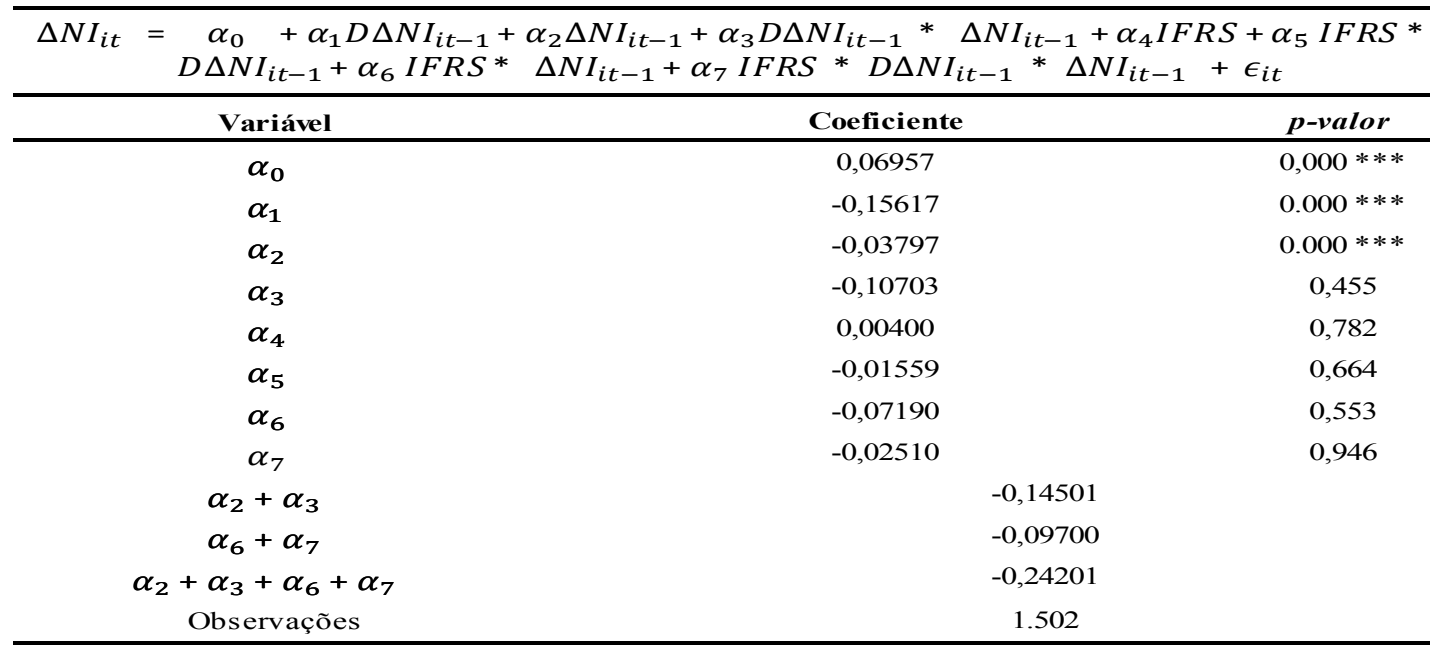

Nota. Método de estimação: Dados em Painel - Modelo Pooled, com a utilização do Erro Padrão Robusto; e são as variações do lucro das Sociedades de Economia Mista, escalonadas pelo valor de seus ativos no início do ano; é uma variável dummy que assume o valor 1 , se <0, e valor 0 , caso contrário; * é uma variável de interação que capta os efeitos de variações negativas antecedente; é uma variável dummy que assume o valor 1 para resultados das Sociedades de Economia Mista em períodos pré convergência às normas do IFRS (anos de 1997 a 2007) e valor 0 para os resultados das Sociedades de Economia Mista no período pós convergência (anos de 2008 a 2014); * é uma variável que capta o intercepto do modelo; * é uma variável de interação que capta o efeito de variações antecedentes nos resultados das Sociedades de Economia Mista em períodos pós convergência; * * é uma variável discriminatória para os valores referentes as Sociedades de Economia Mista em períodos pós convergência; é o termo de erro do modelo.

$*, * *$ e *** denotam significância estatística a $10 \%, 5 \%$ e $1 \%$, respectivamente. 
A Tabela II apresenta os resultados do constructo de conservadorismo e especificamente no coeficiente indica se existe diferença entre o conservadorismo nas SEM no período pré-adoção das IFRS e no período pós-adoção. Como o resultado deste coeficiente não apresentou significância estatística não é possível afirmar que a adoção das IFRS trouxe alguma melhora na qualidade da informação contábil, sob o enfoque do constructo do conservadorismo para o resultado reportado das Sociedades de Economia Mista, permanecendo com o mesmo nível de qualidade verificado no período pré-adoção das IFRS, demonstrando que as SEM não tendem a apresentar reconhecimento oportuno das perdas, que é o comportamento esperado quando existe conservadorismo condicional nas entidades.

\section{Considerações Finais}

As Sociedades de Economia Mistas compõem um grupo de empresas que muito raramente são estudadas pela ciência contábil de modo exclusivo, pois além de possuírem um quantitativo reduzido de empresas de capital aberto (grupo de empresas no quais se desdobram a maioria das pesquisas vinculadas à qualidade da informação contábil), sua diversidade de ramos de atividade também as torna um impeditivo para aprofundamento de suas características.

A proposta do estudo foi investigar esse tipo empresarial muito peculiar da atividade empresarial brasileira, que possui relevância econômica haja vista que algumas grandes empresas brasileiras são enquadradas nesta forma de constituição empresarial e que comumentemente são estudadas em conjunto com outros tipos empresariais.

A questão, de forma simplificada, era determinar se os atributos de qualidade na informação contábil divulgada pelas SEM sofreram alteração após a adoção das normas internacionais de contabilidade pelas empresas brasileiras.

Em relação a esta convergência da contabilidade brasileira às normas internacionais, foi observado que, no caso das Sociedades de Economia Mista, não houve grandes impactos (estatisticamente falando) na qualidade da informação reportada por essas empresas, conforme as análises realizadas, que denotam que a Persistência dos Resultados apresentou resultados mais persistentes nos períodos pós-convergência do que os apresentados nos períodos pré-convergência; já no caso do Conservadorismo Condicional, não se apresentou nenhum resultado significativo de diferenças entre os períodos de pré e pós-convergência.

A não alteração significativa dos constructos de qualidade da informação contábil após a convergência ao IFRS está em concordância com as conclusões dos estudos de Sousa, Souza e Demonier (2016); Santos e Cavalcante (2014) e Castro et.al (2015), que também atestaram não haver alteração significativa na qualidade da informação contábil reportada após a adoção dos IFRS.

Estes resultados, em conjunto, denotam que o processo de convergência da contabilidade brasileira para a normas internacionais não impactou de forma significativa para um aumento da qualidade da informação contábil das Sociedades de Economia Mista, resultado semelhante ao verificado em outros tipos empresariais, o que pode significar que a contabilidade brasileira já apresentava alto grau de qualidade informacional e a alteração para os novos padrões não alterou, de forma significativa, a qualidade descrita.

Para futuras pesquisas sugere-se inserir novos constructos para verificar a qualidade da informação contábil e analisar as eventuais alterações após a convergência contábil para às IFRS no Brasil. 


\section{REFERÊNCIAS}

BALL, R.; SHIVAKUMAR, L. Earnings quality UK private firms: comparative loss recognition timeliness. Journal of Accounting and Economics, v. 39, p. 83-128, 2005.

BALTAGI, B. H. Econometric Analysis of Panel Data. 4. ed. Chichester, United Kingdom: Wiley \& Sons, 2008.

BASU, S. The conservatism principle and the asymmetric timeliness of earnings. Journal of Accounting and Economics, v. 24, p. 03-37, 1997.

BEAVER, W.H.; RYAN, S.G. Conditional and Unconditional Conservatism: Concepts and Modeling. Review of Accounting Studies, v. 10, n. 2-3, p .269-309, 2005.

BIN, D.; CASTOR, B.V.J. A sociedade de Economia Mista e a (I)Lógica da Dualidade EstatalPrivado. In XXXI ENANPAD, Rio de Janeiro: Associação Nacional dos Programas de PósGraduação e Pesquisa em Administração, 2007.

BONFIM, N. B. O interesse público nas Sociedades de Economia Mista. Dissertação de Mestrado, Faculdade de Direito, Universidade de São Paulo, São Paulo, 2011.

BU, D.; ZHANG, C; LIN, P.T.; HU, F. Political uncertainty, institutions, and accounting conservatism: Evidence from the provincial official turnover in China. Spanish Journal of Finance and Accounting, v. 49, n. 4, p. 395-426, 2020. Recuperado em 15 de dezembro de 2020, de https:// www.tandfonline.com/doi/pdf/10.1080/02102412.2019.1657551.

CASTRO, L.A.; PONTE, V.M.R.; COELHO, A.C.; OLIVEIRA, M.C. Padrões Contábeis e Qualidade Informacional: Evidências para o Brasil. Revista Contabilidade Vista e Revista, v. 26, n. 3, p. 124-145, 2015.

COELHO, A. C. D. Qualidade informacional e conservadorismo nos resultados publicados no Brasil. Tese de Doutorado, Faculdade de Economia, Administração e Contabilidade, Universidade de São Paulo, São Paulo, 2007.

CPC - Comitê de Pronunciamentos Contábeis. Pronunciamento Técnico CPC 00 (R2) - Estrutura Conceitual para Relatório Financeiro. Recuperado em 15 de dezembro de 2020, de https://static.cpc. aatb.com.br/Documentos/573_CPC00(R2).pdf

DAI, L.; NGO, P.T.H. Political Uncertainty and Accounting Conservatism: Evidence from the U.S. Presidential Election Cycle, 2013. Working Paper. Recuperado em 13 de dezembro de 2020, de https://mpra.ub.uni-muenchen.de/44283/

DAMASCENA, L.G.; DUARTE, F.C.L.; PAULO, E; Meta-Análise dos Efeitos da Adoção das IFRS na Qualidade da Informação Contábil no Brasil. Revista de Contabilidade do Mestrado em Ciências Contábeis da UERJ (online), Rio de Janeiro, v. 22, n. 1, p. 28-48, 2017.

DECHOW, P.M.; GE, W.; SCHRAND, C.M. Understanding earnings quality: A review of the proxies their determinants and their consequences. Journal of Accounting and Economics, v. 50, p. 344401, 2010.

DECHOW, P.M.; SCHRAND, C.M. Earnings Quality. The Research Foundation of CFA Institute: United States of America, 2004.

DECHOW, P.M.; SKINNER, D.J. Earnings management: reconciling the views of accounting academics, practitioners, and regulators. Accounting Horizons, v. 14, n. 2, p. 235-250, 2000. 
EWERT, R.; WAGENHOFER, A. Earnings management, Conservatism, and Earnings Quality. Foundation and Trents ${ }^{\circledR}$ in Accounting, v. 6, n. 2, p. 65-186, 2011.

FASB - Financial Accounting Standards Board. Statement of concepts n⿳0 02: Qualitative characteristics of accounting information. Stamford, CT: FASB, 1975.

FÁVERO, L. P. L.; BELFIORE, P. P.; CHAN, B. L.; SILVA, F. L. Análise de dados: modelagem multivariada para tomada de decisões. Rio de Janeiro: Campus, 2009.

FERREIRA, L. V.; LEMES, S.; FORTI, C. A. B. Qualidade informativa do lucro líquido e do lucro abrangente. Enfoque Contábil, v. 34, n. 3, p. 1-20, 2015

GUJARATI; D. N. Econometria básica. 4. ed. Rio de Janeiro: Elsevier, 2006.

GUJARATI, D. N.; PORTER, D. C. Econometria Básica. 5. ed. Porto Alegre: AMGH, 2011.

LOPES, A.B. A informação contábil e o mercado de capitais. São Paulo: Pioneira Thomson Learning, 2002.

MELLO, C.A.B. Sociedades Mistas, Empresas Públicas e o Regime de Direito. Revista Eletrônica de Direito Administrativo Econômico, v.10, maio/jul./2007. Recuperado em 22 de junho de 2020, de www.direitodoestado.com.br/codrevista.asp? $\operatorname{cod}=197$

MORAES, A. Constituição do Brasil Interpretada e Legislação Constitucional. São Paulo: Atlas, 2002

MOST, K. S. Accounting Theory. Columbus, OH: Grid Publishing Ind, 1982.

PALEPU, K.G.; HEALY, P.M.; BERNARD, V.L. Business analysis and valuation: using financial statements. 3. ed. Ohio: Thomson Learning, 2004.

PAULO, E.; ANTUNES, M.T.P.; FORMIGONI, H. Conservadorismo contábil nas companhias abertas e fechadas brasileiras. Revista de Administração de Empresas, v. 48, n. 3, p. 46-60, 2008.

RODRIGUES, J.M. Convergência contábil internacional: uma análise da qualidade da informação contábil em razão da adoção dos padrões internacionais de contabilidade emitidos pelo IAS. Tese de Doutorado, Programa Multiinstitucional e Inter-Regional de Pós-Graduação em Ciências Contábeis - UnB/UFPB/UFRN. Brasília, 2012.

RUCKERT, I.N.J. Alguns Aspectos das empresas estatais no Brasil. Ensaios FEE, v. 2, n. 1, 75-93, 1981.

SANTOS, M.A.C.; CAVALCANTE, P.R.N. O efeito da adoção dos IFRS sobre a relevância informacional do lucro contábil no Brasil. Revista Contabilidade \& Finanças, v. 25, n. 66, p. 228$241,2014$.

SCHIPPER, K.; VINCENT, L. Earnings Quality. Accounting Horizons, v. 17, p. 97-10, 2003.

SILVA, A.; BRIGHENTI, J.; KLANN, R.C. Efeitos da Convergência às Normas Contábeis Internacionais na Relevância da Informação Contábil de Empresas Brasileiras. Revista Ambiente Contábil, v. 10, n. 1, p. 121-138, 2018.

SILVA, J.S.; BONFIM, M.P.; NIYAMA. J.K.; SILVA, C.A.T. Adoção ao Padrão IFRS e Earnings Quality: a persistência do lucro das empresas listadas na BM\&FBOVESPA. Revista de Controladoria e Organizações, v. 11, n. 29, p. 46-55, 2017.

SLOAN, R. Do stock prices fully reflect information in accruals and cash flows about earnings? The accounting review, v. 71, p. 289-315, 1996.

SOUSA, E.F.; SOUSA, A.F.; DEMONIER, G.M. Adoção do IFRS no Brasil: Efeitos no Conservadorismo Contábil. Revista de Educação e Pesquisa em Contabilidade, v. 10, n. 2, p. 136$147,2016$. 
STERLING, R. R. Theory of the measurement of enterprise income. Lawrence: University Press of Kansas, 1970.

WATTS, R.L. Conservatism in accounting part I: explanations and implications. Accounting

Horizons, v. 17, n. 3, p. 207-221, 2003. 